\title{
De l'organisation narrative à l'organisation argumentative : les effets paradoxaux d'un référentiel qualité
}

Youcef Aïssani et Odile Bordes

\section{(2) OpenEdition \\ Journals}

Édition électronique

URL : http://journals.openedition.org/communicationorganisation/477

DOI : 10.4000/communicationorganisation.477

ISSN : $1775-3546$

Éditeur

Presses universitaires de Bordeaux

Édition imprimée

Date de publication : 1 juin 2008

Pagination : 72-81

ISBN : 978-2-86781-506-5

ISSN : 1168-5549

Référence électronique

Youcef Aïssani et Odile Bordes, « De l'organisation narrative à l'organisation argumentative : les effets paradoxaux d'un référentiel qualité », Communication et organisation [En ligne], 33 | 2008, mis en ligne le 01 juin 2011, consulté le 20 avril 2019. URL : http://journals.openedition.org/

communicationorganisation/477 ; DOI : 10.4000/communicationorganisation.477 
Dossier : Conduire le changement organisationnel?

\title{
Résumé
}

A ce jour, peu de recherches ont étudié les effets du management de la qualité sur les rationalités narrative et argumentative. Cette étude a pour objectif de montrer que la mise en œuvre collective de la démarche qualité favorise le développement d'une structure narrative. Inversement, l'introduction des principes de la qualité selon le style managérial «top-down» génère un phénomène de résistance au changement et une structure argumentative.

\section{Mots-clés}

Changement organisationnel, management de la qualité, référentiel, implication, établissement médico-social

\begin{abstract}
To date little research has examined the effects of the quality management on the narrative rationality and the argumentative rationality. The purpose of this study is to show that the collective quality management implementation contributes to the development of narration. Conversely, the implementation of the quality principles in accordance to the top-down management style generates a "resistance to change" phenomenon and argumentative rationality.
\end{abstract}

\section{Key-Words}

Organizational change, quality management, systeme of reference, involvement, health centre

\section{Youcef AÏSSANI}

Youcef Aïssani est maître de conférences à l'Université Paul Sabatier - Toulouse III (IUT Tarbes). Il y enseigne la communication et la psychosociologie. Il est co-responsable de l'équipe Changements Organisationnels et Qualité au LERASS.

\section{Odile BORDES}

Odile Bordes est maître de conférences à l'Université Paul Sabatier Toulouse III (IUT A). Elle y enseigne la communication, la qualité des services et la psychosociologie. Elle est membre du Lerass; ses travaux portent sur les effets organisationnels de la démarche qualité. 


\section{De l'organisation narrative à l'organisation argumentative : les effets paradoxaux d'un référentiel qualité}

Youcef Aïssani, Odile Bordes

youcef.aissani@iut-tlse3.fr

odile.bordes@iut-tlse3.fr

Selon Weick, les organisations peuvent se répartir en deux grandes catégories selon qu'elles procèdent d'une logique narrative ou argumentative. Cette distinction permet de classer les organisations sur la base de critères de communication. Aujourd'hui largement admise, elle soulève la question, à ce jour relativement peu explorée, du passage d'une forme à l'autre. Dans le cadre de cette étude, nous nous emploierons à cerner le déterminant et les modalités du changement organisationnel. Après avoir rappelé la définition de ces types d'organisation, nous montrerons l'intérêt d'aborder la question en se plaçant au croisement de deux perspectives : le paradigme de l'instrument de gestion et l'approche pragmatique de Weick. Enfin, nous présenterons les résultats de notre investigation empirique centrée sur les effets paradoxaux d'un outil de gestion en vogue : le référentiel qualité.

\section{Organisations narrative et argumentative}

La mouvance interactionniste dans laquelle l'œuvre de Weick s'inscrit a conduit l'auteur à déployer une réflexion critique des organisations. Contre l'approche holiste et réifiante de l'organisation, longtemps dominante, Weick développe une perspective centrée sur les microprocessus communicationnels qui font la vie organisationnelle. Il considère que l'organisation se confond avec l'ensemble des interactions réciproques. La communication ne se ramène donc nullement à un sous-système de l'organisation, comparable aux autres sous-systèmes que sont la production, la finance, les ressources humaines, et pas davantage à un dispositif de gestion destiné à l'amélioration de la performance globale. Elle est fondatrice de l'ordre organisationnel (Giordano, 2006). Considérant ainsi que l'architecture globale d'une organisation découle des caractéristiques des 
communications qu'y tissent les acteurs, Weick et Browning (1986) proposent de distinguer deux formes majeures d'organisation. Dans l'organisation argumentative, le discours de la rationalité et l'instrumentation de gestion sont érigés en principes organisateurs de l'action collective. L'organisation est appréhendée comme un tout homogène, constitué d'activités articulées entre elles selon des dispositifs et des règles rationnels. Dominée par une logique formelle et fonctionnelle (description des procédures à suivre, élaboration d'instruments de contrôle, rappel des objectifs à atteindre, discours de légitimation...), la communication se déploie sur un mode centralisé intégré à la hiérarchie. Dans l'organisation narrative d'autre part, l'accent est mis davantage sur les interactions interpersonnelles et les échanges entre services médiatisés par les acteurs. On a affaire, ici, à une communication riche en «histoires » (Browning) et en narrations, qui permet l'expression des multiples expériences individuelles ou groupales où s'entremêlent des contenus affectifs, imaginaires et des références au réel. Contrairement au discours argumentatif, les narrations opèrent sur un mode décentralisé appelant dialogue et participation. Ce type d'organisation favorise l'émergence de spécificités locales (normes et attitudes de groupe, communication ritualisée, formation de cultures de service...) et le développement de logiques d'action différenciées.

\section{Instrumentation de gestion et changement organisationnel}

A nos yeux, la valeur heuristique de cette distinction (qui n'est pas sans rappeler l'opposition entre les organisations mécaniques et organiques proposée par Burns et Stalker, 1961) ne se réduit pas à sa vertu taxonomique. Nous pensons en effet qu'elle peut s'étendre à la problématique du changement. Précisément, la typologie de Weick et Browning nous paraît contenir en germe la question du passage d'une forme organisationnelle à l'autre. Une organisation narrative peut-elle évoluer vers une forme argumentative (et vice versa) ? Selon quelles modalités? Quels peuvent être les dispositifs ou mécanismes déclencheurs d'une telle dynamique ? Quelle place les acteurs peuventils y occuper ? Ces questions, nous les formulerons et nous les traiterons dans le cadre de ce qui semble se présenter aujourd'hui comme le paradigme de l'instrumentation de gestion. Destinés au recueil et à l'analyse comparative, prévisionnelle et rétrospective des informations relatives au fonctionnement organisationnel, les outils de gestion doivent permettre la mise en place de l'action collective organisée, ainsi que le pilotage de la performance. Toutefois, par la problématique sociale et communicationnelle qu'ils soulèvent, ils 
dépassent largement le domaine comptable et financier, d'où ils tirent pourtant leur origine. L'idée que les outils de gestion (parmi lesquels les tableaux de bord, le reporting, la planification PERT, le ROI, les ERP...) sont porteurs de changement est largement admise, bien que des désaccords subsistent aujourd'hui encore sur les mécanismes impliqués. Nous examinerons deux points de vue. Selon la perspective gestionnaire (Moisdon, 1997; Bancel-Charansol et Jougleux, 2004), fortement teintée de fonctionnalisme, le pouvoir structurant des outils de gestion peut revêtir quatre formes principales. La première correspond à leur capacité à normer le comportement des acteurs en vue d'atteindre un optimum, la plupart du temps défini par la hiérarchie. La seconde renvoie à leur aptitude à révéler des dysfonctionnements organisationnels, réels ou perçus, et à suggérer des axes d'améliorations possibles (référentiels qualité, tableau de bord...). Par ailleurs, bien que n'intervenant pas comme catalyseur direct du changement, certains outils agissent en tant que support d'accompagnement de la mutation, envisagée et planifiée avant même l'implantation de l'outil. Celui-ci doit permettre la mise en œuvre du changement en facilitant le travail de mise en commun des représentations. Précisons que l'élaboration de la vision partagée ne concerne pas la totalité de l'organisation : elle se cantonne aux acteurs qui appartiennent à un même secteur et utilisent le même outil. Enfin, par le capital de connaissances nouvelles qu'il introduit, l'outil de gestion peut conduire à une mise en cause des savoirs techniques existants. Révélant l'insuffisance des pratiques et des méthodes antérieures, il suscite le besoin de développer des modes opératoires plus adaptés. Aussi est-il à même de déclencher un processus d'apprentissage organisationnel.

\section{Vers une approche pragmatique de l'impact de l'instrumentation de gestion}

L'approche gestionnaire a le mérite de mettre au jour la diversité des mécanismes de changement, mais l'inconvénient d'évacuer la part sociale et non quantifiable de l'outil. Elle tend à reléguer à l'arrièreplan les logiques de l'acteur, passant sous silence le fait que la conception, la mise en place et l'utilisation de l'instrument restent engluées dans des relations de pouvoir. Les changements organisationnels observés ne découlent pas des seules caractéristiques techniques de l'outil, ils dépendent aussi de ses modalités d'insertion dans les rapports sociaux qui fondent la vie organisationnelle (Lorino, 2007). Tout autrement se présente la position - résolument pragmatique de Weick. Récusant la conception de l'outil comme système de modélisation du réel, érigé en guide objectif pour l'action et la décision, 
Dossier : Conduire le changement organisationnel?

l'auteur invite à le (re)considérer sous un angle psychologique et comme un acte de langage. En tant qu'objet d'investissement affectif et foyer de cristallisation des imaginaires, l'outil de gestion a des répercussions sur la motivation des acteurs, facilitant ainsi ou entravant l'action collective. En tant que grille de lecture et de décodage de la « réalité » organisationnelle (informations comptables, comportements, prévisions), qui donne à voir certains aspects de l'organisation (et qui en occulte d'autres), il agit doublement. En premier lieu, il diffuse auprès de ses utilisateurs un nouveau langage et par conséquent, de nouvelles façons de penser l'organisation. En second lieu, et en particulier quand il s'inscrit dans une logique de domination et d'influence (et de résistance), il oriente le contenu et les modalités de l'interaction. C'est dans ce contexte que s'inscrit notre investigation. En réaction à l'approche trop étroitement gestionnaire de l'outil de gestion, en accord avec l'optique psychosociologique et pragmatique de Weick, nous admettrons que le pouvoir structurant de l'instrument dépend moins de son architecture interne que des conditions de sa réception auprès de ses utilisateurs (perception, attitude, valeurs locales...). Nous nous proposons d'étudier comment, en fonction du mode d'appropriation de l'outil, les acteurs développent à son égard une attitude positive ou négative, laquelle à son tour peut orienter les communications vers un modèle narratif ou argumentatif. Plus précisément, on s'attend à ce que dans le cas où l'introduction de l'outil résulte d'une démarche «top-down», les utilisateurs, ayant été écartés de la fixation des objectifs et des plans d'action, assignent à l'outil des significations négativement connotées. Il devrait s'en suivre une réaction de pesanteur communicationnelle, caractéristique de la rationalité argumentative (repli derrière les règles organisationnelles impersonnelles, développement des échanges formels, accentuation des communications hiérarchiques aux dépends des coordinations transversales, tendance à la justification des actes...). Dans le cas contraire d'une démarche «bottom up», qui suppose un style de direction non dirigiste et une organisation faiblement hiérarchisée, on devrait assister au déploiement d'une rationalité narrative. Perçue comme une reconnaissance de l'autre et une incitation à l'expression de soi, cette approche devrait favoriser l'expression de regards croisés sur les différents aspects de la réalité professionnelle. Les discours qui accompagnent la mise en place et l'utilisation de l'outil mêlent le «je » et le «nous», les éléments techniques autant que les vécus des situations de travail nouvelles. 


\section{Présentation de notre investigation empirique et de la méthode retenue}

Le terrain. Nous nous sommes centrés sur le changement organisationnel induit par la mise place de la démarche qualité. Celleci constitue une forme de management qui institue un mode de fonctionnement organisationnel axé sur la satisfaction de l'usager (ou du client) et la qualité des interactions entre ce dernier et les membres de l'organisation. Aux communications verticales et hiérarchiques entre acteurs, elle tend à substituer un modèle d'échanges horizontaux, supposés plus fluides (Pène, 2001; Laurens, 2003). Nous avons sélectionné deux organisations productrices de services à la personne, appartenant au secteur social et médico-social et fortement axées donc sur des activités relationnelles. L'outil de gestion retenu est le référentiel qualité qu'on peut définir comme un document comportant un ensemble d'exigences (règles de conduites, dispositions et modes opératoires) auxquelles l'établissement se doit de répondre en vue de satisfaire les attentes de l'usager (et des instances de certification...). En de nombreux points (taille, public accueilli, statut juridique, ancienneté...) les deux organisations sont comparables. Mais l'une se caractérise par la co-construction du référentiel, impliquant la participation de toutes les catégories du personnel (démarche «bottom-up»); et l'autre, par l'importation par la direction d'un outil prêt à l'emploi, élaboré par un cabinet externe (démarche «op-down»).

Démarche méthodologique ${ }^{l}$. Notre démarche s'appuie sur deux méthodes : la méthode archivistique et l'enquête par entretiens. La première nous a conduit à analyser les documents institutionnels exprimant le discours de l'organisation sur son propre fonctionnement interne, sur sa structure et sur les règles régissant les échanges entre services et entre salariés. L'objectif était de procéder à une évaluation du degré de formalisme visé par l'organisation. Il s'agit pour l'essentiel des référentiels, des comptes-rendus de réunion, du livret d'accueil et du contrat de séjour. L'examen des référentiels devait nous renseigner plus précisément sur les axes de fonctionnement considérés comme prioritaires ainsi que sur les réponses apportées par l'organisation aux situations de non-qualité. D'autre part, les entretiens non directifs devaient nous permettre de mieux cerner les modalités d'appropriation de la démarche qualité par les acteurs

\footnotetext{
${ }^{1}$ Pour plus de précisions, nous renvoyons le lecteur à la présentation originale (Aïssani, 2007).
} 


\section{Dossier : Conduire le changement organisationnel?}

concernés. Afin de pouvoir mettre en évidence les effets de la mise en œuvre de la politique qualité, nous avons programmé les entretiens neuf mois après l'introduction du référentiel. Les réponses ont été soumises à une analyse de contenu.

\section{Résultats et conclusion}

Les salariés (condition «top-down») évoquent spontanément les réunions, aussi importantes que nombreuses, qui ont ponctué la démarche. Lieu d'informations et de débats, elles ont permis à chaque intervenant d'interpeller les pratiques des collègues, de questionner le fonctionnement de l'établissement, de faire part de ses préoccupations personnelles ou d'équipe et d'interroger les résidents. Nombre de ces réunions nous sont apparues comme des espaces de mise en scène collective d'évènements passés ; les participants ont pu témoigner de leurs expériences, restituer sous forme de récits des évènements significatifs au plus près de la réalité vécue. Les séances consacrées au repérage des domaines à inclure dans le référentiel (par exemple : rapports à la famille de l'usager, procédures d'admission, vie sociale, partenariat et réseaux...) et à la définition des indicateurs ont été particulièrement éprouvantes. Elles ont en effet impliqué d'importants efforts d'imagination et de réflexion sur sa propre activité. Mais surtout, elles ont parfois donné lieu à des échanges conflictuels, à des stratégies de négociation, chaque catégorie professionnelle craignant une sous-représentation dans le référentiel. La dynamique communicationnelle et sociocognitive enclenchée par les réunions qualité a été relayée par d'autres dispositifs institutionnels (boîte à idées, enquête par questionnaire auprès des résidents). Fait intéressant, elle s'est déplacée vers d'autres espaces de communication - les échanges informels de tous les instants, les discussions de couloir.

Nous avons procédé à l'analyse de plusieurs types de documents (comptes rendus de réunion, livret d'accueil, contrat de séjour, cahier de liaison) afin d'étudier le type de réponse apportée par l'établissement aux situations de non qualité. Il peut s'agir, là, par exemple de fautes, de manquements aux règles en vigueur avec des degrés divers de gravité, imputés par le professionnel à l'usager ou l'inverse. La comparaison entre les deux structures révèle des particularités de fonctionnement particulièrement intéressantes. L'établissement qui a mis au point le référentiel qualité semble ne pas avoir prévu de procédure organisée et systématique. Si le débat contradictoire est prévu, les situations conflictuelles sont traitées «sur le tas » ou dans le bureau du directeur, sur le mode de l'explication et de la négociation. On peut voir là une marque de la culture orale, 
caractéristique du secteur médico-social. Dans l'autre établissement en revanche, la réponse aux situations litigieuses se fait dans les règles et selon la procédure hiérarchique. La position légaliste de l'institution et de la direction s'exprime nettement dans le souci d'expliciter par écrit les voies de recours possibles, les sanctions encourues...

La comparaison des deux référentiels met au jour des orientations contrastées qui vont dans le sens des résultats précédents. Le référentiel «importé » se singularise par l'accent mis sur les questions relatives au management de l'établissement, à son fonctionnement formel, à l'organisation. Celui qui a été élaboré collectivement associe les thèmes de la communication, du bien-être et de la socialisation des résidents. L'utilisation répétée de ces référentiels dans le cadre de la politique d'amélioration continue de la qualité va amener les acteurs à se décentrer des activités non valorisées, voire ignorées, par le référentiel. Les travailleurs sociaux tendront à développer des pratiques orientées vers la relation à l'usager (souci d'une qualité de la relation de service) ou vers le bon fonctionnement de la structure (souci d'une qualité des processus organisationnels).

L'ensemble des résultats obtenus suggère avec force l'existence de deux logiques de fonctionnement. D'une part, on voit émerger et se développer sous l'effet d'une co-construction d'un référentiel une organisation narrative. On voit aussi le rôle essentiel que les réunions jouent dans cette formation. On rejoint en cela la position de Weick qui, comme le rappelle Giordano (2006), «encourage [...] les réunions, [elles] constituent une miniature de l'organisation; elles sont les briques qui forment la réalité organisationnelle, en quelque sorte un laboratoire de l'élaboration du sens» (p. 164). Cette pratique, qui pourrait être généralisée à l'ensemble des outils de gestion, permettrait de limiter les dysfonctionnements organisationnels et humains provoqués par l'irruption brutale d'une instrumentation de gestion étrangère à la culture locale. D'autre part, et inversement, l'adoption unilatérale de l'outil, peu soucieuse d'accompagner la démarche par une politique de communication, tend à se solder par une perversion du dispositif : le référentiel devient un but en soi, le respect des règles, un phénomène résiduel qui vient parasiter la relation à l'usager. La rationalité argumentative qui prend place progressivement peut être interprétée comme un phénomène organisationnel réactionnel, voire comme une forme de résistance à l'ordre gestionnaire, et à ses piliers que sont l'évaluation, le contrôle et la quantification. Nous pensons que la distinction entre l'organisation narrative et l'organisation argumentative ne saurait se réduire à une opposition entre deux «styles» de communication 
Dossier : Conduire le changement organisationnel?

organisationnelle. Le passage de l'une à l'autre gagnerait à être pensé au travers de la problématique du pouvoir.

\section{Bibliographie}

AISSANI, Youcef. Référentiel qualité et structure de communication. Document manuscrit, 2007, p. 1-22.

AISSANI, Youcef, BORDES Odile. Démarche qualité, sensemaking et émergence dans des structures de communication. Revue Internationale de Psychosociologie, 2007, n 29, p. 59-90.

ALTER, Norbert. L'innovation ordinaire. Paris : Presses Universitaires de France, 2000, 278 p.

BANCEL-CHARENSOL, Laurence, JOUGLEUX, Muriel. L'impact des outils de gestion sur le travail en «front office». Travail et Emploi, 2004, n99, p. 103-119.

BURNS, Tom, STALKER, Georges M. The management of innovation. London : Tavistock, 1961.

LAURENS, Patrick. L'impensable communication dans les normes de système de management de la qualité. Communication et Organisation, 2003, n 23 , p. 135-150.

GIORDANO, Yvonne. S'organiser c'est communiquer : le rôle fondateur de la communication dans l'organizing chez Karl E. Weick. In : DAUTISSIER, J.F. et BENSEBAA, F. Coord. Les défis $d u$ sensemaking en entreprise. Paris : Economica, 2006, p. 153-168.

LORINO, Philippe. Un défi pour les sciences de gestion : le tournant paradigmatique du modèle de la décision au modèle de l'activité collective. In : MARTINET, A.C. Coord. Sciences du management : épistémique, pragmatique et éthique. Paris : Vuibert, 2007, p. 69-84.

MOISDON, Jean-Claude, coord. Du mode d'existence des outils de gestion. Paris : Seli Arslan. 1997. 286 p.

PENE, Sophie. Les agencements langagiers de la Qualité. In : BORZEIX, A. et FRAENKEL, B. Coord. Langage et Travail : communication, cognition et action. Paris : CNRS Editions, 2001, p. 303-322.

TAYLOR, James R., The communicational basis of organization, between the conversation and the text. In : PUTMAN, L.L et KRONE, K.K. coord., Handbook of organizationnal communication. London : Sage, 2006. 
De l'organisation narrative à l'organisation argumentative

WEICK, Karl E. Making sense of the organization. Oxford: Maiden Blackwell. 2001, 323 p.

WEICK, Karl E., BROWNING Larry D., Argument and narration in organizational communication. Journal of Management, 1986, $\mathrm{n}^{\circ} 12$, p. 243-259. 
